\title{
Diffusion Processes with Singular Drift Fields
}

\author{
Philippe Blanchard and Simon Golin * \\ Department of Physics and Research Centre Bielefeld-Bochum-Stochastics, \\ University of Bielefeld, D-4800 Bielefeld 1, Federal Republic of Germany
}

\begin{abstract}
A class of stochastic differential equations with highly singular drift fields is considered. Using a purely probabilistic approach, we can show the unattainability of the nodal set. Moreover, a global existence and uniqueness theorem for diffusion processes with singular drift fields is established. The finite action condition of Carlen and Zheng can be modified. We relate our results to the diffusions which describe the time evolution of quantum systems in stochastic mechanics.
\end{abstract}

\section{Introduction and Summary}

The main bulk of the mathematical literature on stochastic differential equations (SDEs) tackles the question of existence and uniqueness of solutions under assumptions very reminiscent of those for deterministic (ordinary or partial) differential equations (see e.g. [1-3]). Usually the coefficients of the SDEs (the socalled infinitesimal characteristics) are required to satisfy some regularity condition (such as a Lipschitz condition) to ensure local existence and uniqueness of a continuous solution, and a growth condition is imposed to avoid explosions, i.e. to prevent the process from running off to infinity within finite time.

Both from a mathematical point of view and a look towards applications in other disciplines, e.g. physics or biology, it is desirable to relax the standard conditions.

There has been some previous work in this field. The stationary case was first considered by Albeverio and Høegh-Krohn [4], and then by Carmona [5], Nagasawa [6], and Albeverio et al. [7]. The analysis in [5,7] is in terms of Dirichlet forms. A nice feature of this work is that the probability densities involved are admitted to be discontinuous. The point of view of Dirichlet forms is also taken in papers by Albeverio et al. [8], and Fukushima [9]. Reference [8] also discusses several physical aspects of the unattainability of the nodal set (i.e. the set

\footnotetext{
* Present address: Technische Universität Berlin, Fachbereich Mathematik MA 7-2, Strasse des 17. Juni 136, D-1000 Berlin 12
} 
where the probability density vanishes), viz. natural phenomena such as the formation of planetary systems, the morphology of galaxies, the formation of zones of winds in the atmosphere, and the formation of spokes in the rings of Saturn. Nagasawa [6] presents a model of segregation of a population in an environment, which is of interest in some applications to biology and ecology. In a recent paper by Blanchard and Zheng [10] the stationary case is dealt with by employing a pathwise conservation law.

The non-stationary situation was solved only recently. In the case of a compact manifold this was accomplished by Nelson [11,12]. But the compactness condition can be dropped. For a Euclidean configuration space Carlen established weak existence of solutions of stochastic differential equations with singular drifts by means of PDE methods [13]. In a completely different spirit, Zheng used tightness results for semi-martingales to obtain weak existence for diffusions on a Riemannian configuration space [14]. This probabilistic approach has the advantage of exhibiting explicitly that the diffusions avoid the nodes. On the other hand, Carlen's analysis requires less regularity. Both Carlen and Zheng use a kind of (global) finite action condition to construct the diffusions. In fact, Zheng and Meyer showed [15] that also a local condition guarantees that the diffusions do not reach the nodes in finite time. Finally, by exploiting Carlen's existence proof, Guerra [16] has found a continuity argument to introduce an enlarged class of diffusions with singular drifts.

The work contained in this paper was inspired by $[10,15]$. We use a purely probabilistic approach in terms of suitably defined stopping times. Making use of some physical and geometrical ideas, the construction of diffusions can be carried out in a transparent way. As we shall see, the finite action condition can be modified.

The basic problem to be tackled in the sequel is the following. Suppose we are given at each time $t \in \mathbb{R}_{+}:=[0, \infty)$ a probability density $\varrho(\cdot, t)$ on the $d$-dimensional Euclidean space $\mathbb{R}^{d}$. Define

$$
U:=\left\{(x, t) \in \mathbb{R}^{d} \times \mathbb{R}_{+} \varrho \varrho(x, t)>0\right\}
$$

and the nodal set

$$
N:=U^{c}=\left\{(x, t) \in \mathbb{R}^{d} \times \mathbb{R}_{+} \mid \varrho(x, t)=0\right\} .
$$

Let a drift field $b: U \rightarrow \mathbb{R}^{d}$ be given and suppose that $\varrho$ and $b$ are related to each other by the (forward) Fokker-Planck equation on $U$,

$$
\partial_{t} \varrho=-\operatorname{div}(\varrho b)+v \Delta \varrho,
$$

where $v$ is a positive constant. To make this equation meaningful one needs, of course, some regularity conditions on $\varrho$ and $b$. However, we are only considering a field $b$ which is defined on $U$, i.e. it may be of very singular nature near the boundary of $U$. Suppose we are given a probability space $(\Omega, \mathscr{F}, P)$, a filtration $\left\{\mathscr{F}_{t}\right\}_{t \in \mathbb{R}_{+}}$, and a standard Wiener process $\left\{w_{t}\right\}_{t \in \mathbb{R}_{+}}$adapted to the filtration and with variance $2 v$ (i.e. $\left.E\left[w_{s}^{i} w_{s}^{j}\right]=2 v(s \wedge t) \delta^{i j}(s, t \geqq 0 ; i, j=1,2, \ldots, d)\right)$. Our problem consists in constructing a diffusion process $\left\{X_{t}\right\}_{t \in \mathbb{R}_{+}}$with drift $b$ and probability density $\varrho$, in other words, we will investigate existence and uniqueness of solutions 
of the stochastic differential equation

$$
\left\{\begin{array}{l}
d X_{t}=b\left(X_{t}, t\right) d t+d w_{t}, \\
X_{0}=\eta,
\end{array}\right.
$$

where $\eta$ is a fixed $\mathscr{F}_{0}$-measurable random variable with density $\varrho(\cdot, 0)$. If such a process $X_{t}$ exists then - under suitable conditions - its mean forward derivative (cf. [12]),

$$
\lim _{\Delta t \downarrow 0} \frac{1}{\Delta t} E\left[X_{t+\Delta t}-X_{t} \mid \mathscr{F}_{t}\right]
$$

exists and is equal to $b$, and moreover,

$$
P\left\{f\left(X_{t}\right) \in A\right\}=\int_{A} d x \varrho(x, t) f(x)
$$

for all bounded measurable functions $f$ and all Borel sets $A$. We will remark on the meaning of the equality $b=D X$ in Sect. III.

We now summarize the paper. In Sect. II, we recollect some heuristic ideas underlying the expected behaviour of sample paths of diffusions. The proof of the unattainability of the nodes as well as the global existence and uniqueness theorem is contained in Sect. III. Section IV is devoted to an application of these results to stochastic mechanics. We conclude in Sect. V with some remarks on possible generalizations of the strategy contained in this paper.

\section{Heuristics}

Before going through the mathematical argument it is worthwhile to get some physical intuition about what might prevent a diffusion from being defined globally and why this is not so.

Let us assume for the moment that the SDE (4) has a local solution, i.e. the sample paths of the process $X_{t}$ are defined at least for some finite time interval. But what may go wrong and prevent a trajectory from being defined for all times? This can occur only if the trajectory under consideration approaches the nodes within finite time (and then $b$ is undefined, Fig. 1a) or if it escapes to infinity within the finite interval (Fig. 1b).

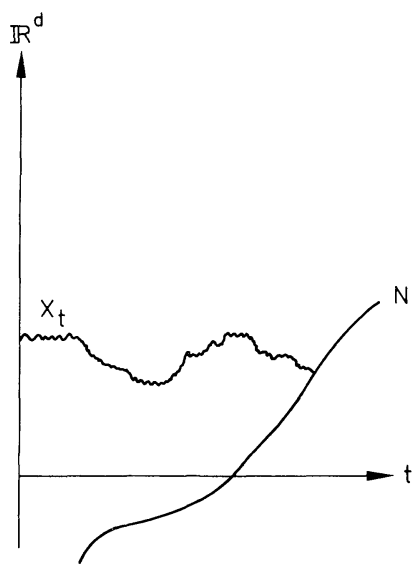

Fig. 1a

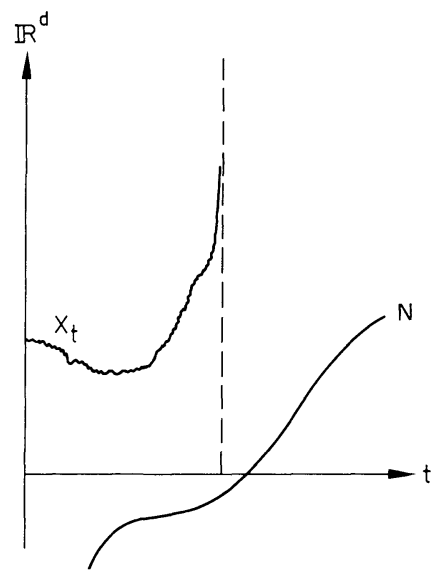

Fig. 1b 
From a physical point of view the unattainability of the nodes seems rather plausible. In the theory of classical diffusions it is not the flow $b$ itself that is of physical significance but the current $\varrho b$, and even if $b$ is singular at the nodes, the product $\varrho b$ may stay finite. In terms of physics this means that hardly any diffusing particle runs into the nodes. Moreover, there could be another mechanism explaining the unattainability of the nodal set: if the singular drift field points away from the nodes it will give rise to a strong repulsion and prevent the particle from approaching the nodes.

In the context of stochastic mechanics the diffusion process is associated to a quantum state (cf. Sect. IV). If $\psi$ denotes the quantum mechanical wave function, then

$$
\begin{gathered}
\varrho=|\psi|^{2}, \\
b=2 v(\operatorname{Re}+\operatorname{Im}) \operatorname{grad} \ln \psi \quad(\text { on } U) .
\end{gathered}
$$

It is easy to check for generic stationary wave functions, e.g., for the bound states of the harmonic oscillator, that the corresponding drifts are repulsive at the nodes, and moreover $\varrho b$ tends to zero.

To prevent explosions (i.e. the escape at infinity) one needs an additional condition [cf. (A.4) in Sect. III]. Again, this will be typically satisfied in the theory of stochastic mechanics.

\section{Unattainability of the Nodes and Global Existence}

In this section we will present our main theorems concerning the construction of diffusion processes with singular drift fields. We do not look for optimal regularity conditions on $\varrho$ and $b$ and as a reward for this we get transparent proofs.

Throughout the sequel we assume that

$$
\begin{gathered}
\varrho \in C^{2,1}\left(\mathbb{R}^{d} \times \mathbb{R}_{+}\right), \\
b \in C^{1,0}(U),
\end{gathered}
$$

and that $\varrho$ and $b$ satisfy the Fokker-Planck equation (3). Here $C^{m, n}$ denotes the set of functions having $m$ continuous spatial and $n$ continuous temporal derivatives. Locally, the drift field $b$ is rather regular, and the standard theorems grant a unique local solution $X_{t}, 0 \leqq t<\zeta$, of the SDE (4) up to the stopping time $\zeta$ (the so-called killing time). In fact, existence and uniqueness are in the strong sense.

We will need the following lemma [15]. It relates the process $X_{t}$, which has possibly been stopped (if $\zeta<\infty$ ), to the globally defined density $\varrho$. In the proofs to follow this lemma will be a key ingredient.

Lemma 1. Let $f(x, t)$ be a non-negative function and let $\tau$ be a random variable satisfying $0 \leqq \tau \leqq \zeta \wedge k$ for some $k>0$. Then

$$
E\left[\int_{0}^{\tau} d t f\left(X_{t}, t\right)\right] \leqq \int d x \int_{0}^{k} d t \varrho(x, t) f(x, t) .
$$

As E.A. Carlen pointed out to us, the proof of this lemma is an easy consequence of the maximum principle applied to the Fokker-Planck equation (see also $[11,12])$. 
To state our propositions we need some definitions. It is convenient to introduce the osmotic velocity

$$
u:=\left\{\begin{array}{lll}
v \frac{\operatorname{grad} \varrho}{\varrho} & \text { on } & U \\
0 & \text { on } & N
\end{array}\right.
$$

and the current velocity

$$
v:=\left\{\begin{array}{lll}
b-u & \text { on } & U \\
0 & \text { on } & N
\end{array} .\right.
$$

For $y \in(x, t) \in \mathbb{R}^{d} \times \mathbb{R}_{+}$we define the norm

$$
\|y\|:=\sqrt{|x|^{2}+t^{2}}
$$

If $E$ is any subset of $\mathbb{R}^{d} \times \mathbb{R}_{+}$the Euclidean distance between $y$ and $E$ is denoted by

$$
d(y, E):=\left\{\begin{array}{lll}
\min _{y^{\prime} \in E}\left\|y-y^{\prime}\right\| & \text { if } & E \neq \emptyset \\
\infty & \text { if } & E=\emptyset .
\end{array}\right.
$$

where $\emptyset$ is the empty set. Moreover, we put

$$
Y_{t}:=\left(X_{t}, t\right) \text {. }
$$

Theorem 1. Let the process $X_{t}, 0 \leqq t<\zeta$, have a probability density $\varrho$ and $a$ drift $b$ subject to assumptions (A.1) and (A.2). Suppose also that

$$
\varrho \operatorname{div} v \in \mathscr{L}_{\text {loc }}^{1}\left(\mathbb{R}^{d} \times \mathbb{R}_{+}\right) .
$$

Then, for any compact set $K \subset \mathbb{R}^{d} \times \mathbb{R}_{+}$,

$$
P\left\{\left.\omega \in \Omega\right|_{t<\zeta} d\left(Y_{t}, K \cap N\right)>0\right\}=1 .
$$

Proof. Let $k, l \in \mathbb{N}$, and define the compact set

$$
K:=\left\{(x, t) \in \mathbb{R}^{d} \times \mathbb{R}_{+}|| x \mid \leqq l, t \leqq k\right\} .
$$

We also define a subset $A$ of the probability space $\Omega$ by

$$
A:=\left\{\varrho\left(Y_{0}\right)>0, Y_{t} \in K(\forall t<\zeta \wedge k), \inf _{t<\zeta \wedge k} \varrho\left(Y_{t}\right)=0\right\} .
$$

Of course, the set $\left\{\varrho\left(Y_{0}\right)>0\right\}$ has unit probability. Throughout this proof the continuity of the sample paths $t \mapsto Y_{t}$ will be essential. A first consequence of this continuity is the fact that in order to prove the theorem it suffices to show

$$
P(A)=0(\forall k, l \in \mathbb{N}) .
$$

Next we introduce for each $n \in \mathbb{N}$ a stopping time by

$$
T_{n}:=\inf \left\{t<\zeta \wedge k \mid Y_{t} \notin K \text { or } \varrho\left(Y_{t}\right) \leqq \frac{1}{n}\right\}
$$

if $\left\{t<\xi \wedge k \mid Y_{t} \notin K\right.$ or $\left.\varrho\left(Y_{t}\right) \leqq \frac{1}{n}\right\}$ is non-void, and we set $T_{n}:=\zeta \wedge k=k$ otherwise. 
Since the sequence $\left\{T_{n}\right\}$ is non-decreasing we define another stopping time by

$$
T:=\lim _{n} T_{n} .
$$

Clearly, $T>0$ if and only if $\left|Y_{0}\right| \leqq l$ and $\varrho\left(Y_{0}\right)>0$. Thus we may rewrite the set $A$ in terms of stopping times,

$$
A=\left\{T>0, \varrho\left(Y_{T_{n}}\right) \leqq \frac{1}{n}(\forall n \in \mathbb{N})\right\} .
$$

We shall now look at those trajectories for which the density $\varrho\left(Y_{t}\right)$ becomes arbitrarily small or, in other words, $\ln \varrho\left(Y_{t}\right)$ is unbounded from below. An application of Itô's lemma yields

$$
\begin{aligned}
I_{\{T>0\}} \ln \varrho\left(Y_{T_{n}}\right)= & I_{\{T>0\}} \ln \varrho\left(Y_{0}\right)+\int_{0}^{T_{n}} d t\left[\frac{\partial_{t} \varrho}{\varrho}+b \cdot \frac{\operatorname{grad} \varrho}{\varrho}+v \Delta \ln \varrho\right]\left(Y_{t}\right) \\
& +\int_{0}^{T_{n}} d w_{t} \frac{\operatorname{grad} \varrho}{\varrho}\left(Y_{t}\right) \quad(n \in \mathbb{N}),
\end{aligned}
$$

where $I_{(\cdot)}$ is the characteristic function. Now the expectation of each term is to be analyzed.

If $T_{n}>0$ and $t \in\left[0, T_{n}\right]$ then $Y_{t} \in K$, and $\operatorname{grad} \varrho\left(Y_{t}\right)$ is bounded on $K$ by continuity. Moreover, $T_{n}>0$ implies that $\varrho\left(Y_{t}\right) \geqq \frac{1}{n}$ for $t \in\left[0, T_{n}\right]$. Therefore

$$
E \int_{0}^{T_{n}} d t\left|\frac{\operatorname{grad} \varrho}{\varrho}\right|^{2}\left(Y_{t}\right)<\infty
$$

and the stochastic integral $\int_{0}^{T_{n}} d w_{t} \frac{\operatorname{grad} \varrho}{\varrho}\left(Y_{t}\right)$ is a mean zero martingale (indexed by
n) Since $\{T>0\} \subset\left\{\left|Y_{0}\right| \leqq l\right\}$, it follows that

$$
E\left[\left|I_{\{T>0\}} \ln \varrho\left(Y_{0}\right)\right|\right] \leqq E\left[I_{\left\{\left|Y_{0}\right| \leqq l\right\}}|\ln \varrho|\left(Y_{0}\right)\right]=\int d x \varrho(x, 0) I_{\{|x| \leqq l\}}(x)|\ln \varrho|(x, 0) .
$$

The last term in Eq. (23) is finite since $\varrho$ is locally bounded [by (A.1)] and hence $E\left[I_{\{T>0\}} \ln \varrho\left(Y_{0}\right)\right]$ exists and is finite.

Let us consider the left-hand side of (21). If $T>0$ then $Y_{T_{n}} \in K, n \in \mathbb{N}$, and $I_{\{T>0\}} \ln \varrho\left(Y_{T_{n}}\right)$ is bounded from above uniformly in $\omega \in \Omega$ and $n \in \mathbb{N}$. Hence $E\left[I_{\{T>0\}} \ln \varrho\left(Y_{T_{n}}\right)\right]$ is uniformly bounded from above.

Assume now that (17) does not hold, i.e. $P(A)>0$. Our aim is to deduce a contradiction from this assumption. Let us denote the decomposition of realvalued function $f$ into its positive and negative parts by $f=f^{+}-f^{-}$. Since $A$ $C\{T>0\}$ and $\ln \varrho\left(Y_{T_{n}}\right) \leqq-\ln n$ on $A$, it follows that

$$
E\left[I_{\{T>0\}}(\ln \varrho)^{-}\left(Y_{T_{n}}\right)\right] \geqq E\left[I_{A}(\ln \varrho)^{-}\left(Y_{T_{n}}\right)\right] \geqq P(A) \ln n .
$$

As $E\left[I_{\{T>0\}} \ln \varrho\left(Y_{T_{n}}\right)\right]$ is uniformly bounded from above this implies

$$
E\left[I_{\{T>0\}} \ln \varrho\left(Y_{T_{n}}\right)\right] \rightarrow-\infty \text { as } n \rightarrow \infty,
$$


and therefore

$$
E \int_{0}^{T_{n}} d t\left[\frac{\partial_{t} \varrho}{\varrho}+b \cdot \frac{\operatorname{grad} \varrho}{\varrho}+v \Delta \ln \varrho\right]\left(Y_{t}\right) \rightarrow-\infty \text { as } n \rightarrow \infty .
$$

On the other hand, we can rewrite the integrand by means of the FokkerPlanck equation (3);

$$
\frac{\partial_{t} \varrho}{\varrho}+b \cdot \frac{\operatorname{grad} \varrho}{\varrho}=v \frac{\Delta \varrho}{\varrho}-\operatorname{div} b \quad(\text { on } U)
$$

implies

$$
\frac{\partial_{t} \varrho}{\varrho}+b \cdot \frac{\operatorname{grad} \varrho}{\varrho}+v \Delta \ln \varrho=v \frac{\Delta \varrho}{\varrho}-\operatorname{div} v \quad(\text { on } U) .
$$

If $T_{n}>0$ and $t \in\left[0, T_{n}\right]$ then $Y_{t} \in K$, and hence

$$
\begin{aligned}
& E \int_{0}^{T_{n}} d t\left[\frac{\partial_{t} \varrho}{\varrho}+b \cdot \frac{\operatorname{grad} \varrho}{\varrho}+v \Delta \ln \varrho\right]^{-}\left(Y_{t}\right)=E \int_{0}^{T_{n}} d t I_{\left\{T_{n}>0\right\}}\left[v \frac{\Delta \varrho}{\varrho}-\operatorname{div} v\right]^{-}\left(Y_{t}\right) \\
& \quad \leqq E \int_{0}^{T_{n}} d t I_{\left\{Y_{t} \in K\right\}}\left[v \frac{\Delta \varrho}{\varrho}-\operatorname{div} v\right]^{-}\left(Y_{t}\right) \leqq \int d x \int d t I_{K}(x, t)[v \Delta \varrho-\varrho \operatorname{div} v]^{-}(x, t) \\
& \quad \leqq v \iint_{K} d x d t|\Delta \varrho|(x, t)+\iint_{K} d x d t \varrho|\operatorname{div} v|(x, t)
\end{aligned}
$$

where the last but one step follows from Lemma 1. Since $K$ is compact the regularity of $\varrho$ implies that $\iint_{K} d x d t|\Delta \varrho|<\infty$, whereas finiteness of $\iint_{K} d x d t \varrho|\operatorname{div} v|$ follows from assumption (A.3). Thus we have obtained a uniform upper bound for

$$
E \int_{0}^{T_{n}}\left[\frac{\partial_{t} \varrho}{\varrho}+b \cdot \frac{\operatorname{grad} \varrho}{\varrho}+v \Delta \ln \varrho\right]^{-}\left(Y_{t}\right),
$$

and this contradicts (26). As a result we conclude that $A$ cannot have a nonvanishing probability, i.e. (14) is established.

Remark. In the symmetric case, which is defined by $v \equiv 0$, condition (A.3) is trivially satisfied.

In the next theorem it will be proven that explosions in finite time cannot occur, in other words, the process $X_{t}, 0 \leqq t<\zeta$, cannot disappear at infinity within a finite time interval. This follows essentially from the following assumption on the probability density $\varrho$ :

There is no continuous curve $t \mapsto \gamma_{\mathrm{t}}$, defined on some

finite time interval $[0, s)$, such that $\sup _{t<s}\left|\gamma_{t}\right|=\infty$ and $\inf _{t<s} \varrho\left(\gamma_{t}, t\right)>0$.

In addition, we have to strengthen the assumptions on $\varrho$ and its derivatives from a spatially local to a global form. Then this yields a unique global construction of diffusion processes. Here both existence and uniqueness are meant in the strong sense. 
Theorem 2. Let the process $X_{t}, 0 \leqq t<\zeta$, have a probability density $\varrho$ and a drift $b$ subject to assumptions (A.1), (A.2), and (A.4). Suppose also that

$$
\begin{gathered}
\int d x|\Delta \varrho| \in \mathscr{L}_{\mathrm{loc}}^{1}\left(\mathbb{R}_{+}\right), \\
\int d x \varrho|\operatorname{div} v| \in \mathscr{L}_{\mathrm{loc}}^{1}\left(\mathbb{R}_{+}\right),
\end{gathered}
$$

Then

$$
P\{\zeta=\infty\}=1 .
$$

Proof. As in the previous theorem the proof will be accomplished in terms of suitably defined stopping times. For $k, l, n \in \mathbb{N}$ we introduce the stopping times

$$
\begin{gathered}
S^{(1)}:=\left\{\begin{array}{lll}
0 & \text { if } & \left|X_{0}\right|>l \\
\zeta \wedge k & \text { if } & \left|X_{0}\right| \leqq l
\end{array}\right. \\
S_{n}^{(2)}:=\inf \left\{t<\zeta \wedge k \mid \varrho\left(Y_{t}\right) \leqq \frac{1}{n}\right\}
\end{gathered}
$$

if $\left\{t<\zeta \wedge k \mid \varrho\left(Y_{t}\right) \leqq \frac{1}{n}\right\}$ is non-void, and we set $S_{n}^{(2)}:=\zeta \wedge k=k$ otherwise. For technical reasons we define another two families of stopping times,

$$
\begin{gathered}
S_{n}^{(3)}:=\inf \left\{t<\zeta \wedge k|| \operatorname{grad} \varrho \mid\left(Y_{t}\right) \geqq n\right\}, \\
S_{n}^{(4)}:=\inf \{t<\zeta \wedge k \mid \varrho \geqq \ln n\},
\end{gathered}
$$

where $S_{n}^{(3)}$ and $S_{n}^{(4)}$ are set equal to zero if the respective sets on the right-hand side of (33) or (34) are void. By (A.1), the families $S_{n}^{(2)}, S_{n}^{(3)}$, and $S_{n}^{(4)}$ increase monotonically to $\zeta \wedge k$. Thus the sequence

$$
S_{n}:=S^{(1)} \wedge S_{n}^{(2)} \wedge S_{n}^{(3)} \wedge S_{n}^{(4)}
$$

gives rise to the limiting stopping time

$$
S:=\lim _{n} S_{n}=S^{(1)} \wedge \zeta \wedge k .
$$

Clearly, $S>0$ if and only if $\left|X_{0}\right| \leqq l$ and $\varrho\left(Y_{0}\right)>0$.

Proposition (30) follows, in fact, if we could show

$$
P\{\zeta>k\}=1 \quad(\forall k \in \mathbb{N}) .
$$

But we can rewrite the last set [because of (A.4)],

$$
\{\zeta>k\}=\left\{\inf _{t<\zeta \wedge k} \varrho\left(Y_{t}\right)>0\right\} \text {. }
$$

Moreover, it is sufficient to consider trajectories starting within bounded regions around the origin. Since $P\left\{\varrho\left(Y_{0}\right)>0\right\}=1$, it suffices to show that

$$
P(B)=0 \quad(\forall k, l \in \mathbb{N}),
$$

where

$$
B:=\left\{S>0, \inf _{t<\zeta \wedge k} \varrho\left(Y_{t}\right)=0\right\}=\left\{S>0, \varrho\left(Y_{S_{n}}\right) \leqq \frac{1}{n}(\forall n \in \mathbb{N})\right\}
$$


Again, we obtain from Itô's lemma an equation analogous to (21),

$$
\begin{aligned}
I_{\{S>0\}} \ln \varrho\left(Y_{S_{n}}\right)= & I_{\{S>0\}} \ln \varrho\left(Y_{0}\right)+\int_{0}^{S_{n}} d t\left[\frac{\partial_{t} \varrho}{\varrho}+b \cdot \frac{\operatorname{grad} \varrho}{\varrho}+v \Delta \ln \varrho\right]\left(Y_{t}\right) \\
& +\int_{0}^{S_{n}} d w_{t} \frac{\operatorname{grad} \varrho}{\varrho}\left(Y_{t}\right) \quad(n \in \mathbb{N}) .
\end{aligned}
$$

If $S_{n}>0$ and $t \in\left[0, S_{n}\right]$, then $t \in\left[0, S_{n}^{(2)} \wedge S_{n}^{(3)}\right]$ and $\left|\frac{\operatorname{grad} \varrho}{\varrho}\right|\left(Y_{t}\right) \leqq n^{2}$. Therefore

$$
E \int_{0}^{S_{n}} d t\left|\frac{\operatorname{grad} \varrho}{\varrho}\right|^{2}\left(Y_{t}\right) \leqq k n^{4}<\infty,
$$

and the stochastic integral $\int_{0}^{S_{n}} d w_{t} \frac{\operatorname{grad} \varrho}{\varrho}\left(Y_{t}\right)$ is a mean zero martingale.

Since $\{S>0\} \subset\left\{\left|X_{0}\right| \leqq l\right\}$, it follows that

$$
E\left[\left|I_{\{S>0\}} \ln \varrho\left(Y_{0}\right)\right|\right] \leqq E\left[I_{\left\{\left|X_{0}\right| \leqq l\right\}}|\ln \varrho|\left(Y_{0}\right)\right]=\int d x \varrho(x, 0) I_{\{|x| \leqq l\}}(x)|\ln \varrho|(x, 0) .
$$

By (A.1) the density $\varrho$ is locally bounded and hence $E\left[I_{\{S>0\}} \ln \varrho\left(Y_{0}\right)\right]$ exists and is finite.

We now turn to the left-hand side of (41) and use the decomposition

$$
1=I_{\left\{S_{n}=0\right\}}+I_{\left\{S_{n}>0\right\}} .
$$

Since

$$
\begin{aligned}
E\left[I_{\{S>0\} \cap\left\{S_{n}=0\right\}}(\ln \varrho)^{+}\left(Y_{S_{n}}\right)\right] & \leqq E\left[I_{\left\{\left|X_{0}\right| \leqq l\right\}}(\ln \varrho)^{+}\left(Y_{0}\right)\right] \\
& =\int d x \varrho(x, 0) I_{\{x \leqq l\}}(x)(\ln \varrho)^{+}(x, 0) \leqq \mathrm{const}
\end{aligned}
$$

for some finite constant independent of $n$, and

$$
E\left[I_{\{S>0\} \cap\left\{S_{n}>0\right\}}(\ln \varrho)^{+}\left(Y_{S_{n}}\right)\right] \leqq E\left[I_{\left\{S_{n}^{(4)}>0\right\}}(\ln \varrho)^{+}\left(Y_{S_{n}}\right)\right] \leqq \ln \ln n
$$

for $n \geqq 3$, it turns out that $E\left[I_{\{S>0\}} \ln \varrho\left(Y_{S_{n}}\right)\right]$ may not tend to $+\infty$ faster than of the order of $\ln \ln n$.

Let us assume that (39) is not true, i.e. $P(B)>0$. Since $B C\{S>0\}$ and $\ln \varrho\left(Y_{S_{n}}\right) \leqq-\ln n$ on $B$, it follows that

$$
E\left[I_{\{S>0\}}(\ln \varrho)^{-}\left(Y_{S_{n}}\right)\right] \geqq E\left[I_{B}(\ln \varrho)^{-}\left(Y_{S_{n}}\right)\right] \geqq P(B) \ln n,
$$

and this therefore implies

$$
\begin{gathered}
E\left[I_{\{S>0\}} \ln \varrho\left(Y_{S_{n}}\right)\right] \rightarrow-\infty \text { as } n \rightarrow \infty, \\
E \int_{0}^{S_{n}} d t\left[\frac{\partial_{t} \varrho}{\varrho}+b \cdot \frac{\operatorname{grad} \varrho}{\varrho}+v \Delta \ln \varrho\right]\left(Y_{t}\right) \rightarrow-\infty \text { as } n \rightarrow \infty .
\end{gathered}
$$

In analogy with the previous proof we rewrite the integral,

$$
\begin{gathered}
E \int_{0}^{S_{n}} d t\left[\frac{\partial_{t} \varrho}{\varrho}+\right. \\
\left.+b \cdot \frac{\operatorname{grad} \varrho}{\varrho}+v \Delta \ln \varrho\right]^{-}\left(Y_{t}\right) \leqq \int d x \int_{0}^{k} d t[v \Delta \varrho-\varrho \operatorname{div} v]^{-}(x, t) \\
\leqq v \int d x \int_{0}^{k} d t|\Delta \varrho|(x, t)+\int d x \int_{0}^{k} d t \varrho|\operatorname{div} v|(x, t)
\end{gathered}
$$


and these terms are finite according to (A.5) and (A.6). This yields a contradiction to (49), and we conclude that $P(B)=0$.

Remarks. (i) Assumptions (A.5) and (A.6) are to be compared to the finite action condition of Carlen [13] and Zheng [14],

$$
\int d x \varrho\left(u^{2}+v^{2}\right) \in \mathscr{L}_{\text {loc }}^{1}\left(\mathbb{R}_{+}\right) .
$$

Strictly speaking there is no inclusion relation between these conditions, though in a loose sense (A.7) implies (A.5) and (A.6). This can be seen as follows. Provided that no surface term turns up, we have

$$
\int_{U} d x \varrho\left(\frac{\operatorname{grad} \varrho}{\varrho}\right)^{2}=-\int_{U} d x \varrho \operatorname{div} \frac{\operatorname{grad} \varrho}{\varrho} .
$$

Thus the condition $\int d x \varrho u^{2} \in \mathscr{L}_{\text {loc }}^{1}\left(\mathbb{R}_{+}\right)$does not quite imply $\int_{U} d x \varrho\left|\operatorname{div} \frac{\operatorname{grad} \varrho}{\varrho}\right| \in \mathscr{L}_{\text {loc }}^{1}\left(\mathbb{R}_{+}\right)$, which were sufficient for (A.5), since on $U$

$$
\begin{gathered}
\Delta \varrho=\varrho\left[\left(\frac{\operatorname{grad} \varrho}{\varrho}\right)^{2}+\operatorname{div} \frac{\operatorname{grad} \varrho}{\varrho}\right], \\
|\Delta \varrho| \leqq \varrho\left[\left(\frac{\operatorname{grad} \varrho}{\varrho}\right)^{2}+\left|\operatorname{div} \frac{\operatorname{grad} \varrho}{\varrho}\right|\right] .
\end{gathered}
$$

Similarly, $2|u v| \leqq u^{2}+v^{2}$, and thus

$$
\int d x \varrho|u v| \leqq \frac{1}{2} \int d x \varrho\left(u^{2}+v^{2}\right) .
$$

Therefore (provided there is no surface contribution)

$$
\int d x \varrho \operatorname{div} v=\frac{1}{v} \int d x \varrho u v
$$

is in $\mathscr{L}_{\text {loc }}^{1}\left(\mathbb{R}_{+}\right)$by virtue of (A.7). However, (A.6) requires a $|\operatorname{div} v|$ term rather than merely div $v$. In conclusion, our conditions (A.5) and (A.6) constitute a different kind of finite action condition than that of Carlen and Zheng, but often they will be a consequence of (A.7).

(ii) The basic strategy in our proofs is to find appropriate estimates on $\ln \varrho$, and this is done by means of Itô's lemma. In this respect our method is similar to the one employed by Nelson [11,12]. His proof does not require the finite action condition (A.7) but works with the weaker condition $\int_{0}^{k} d t \int d x \varrho|u \cdot v|<\infty$ which,
according to (56), is related to (A.6).

(iii) In [7, Theorem 4.2] (see also [8, Sect. 3]) the unattainability of the nodal set is proven under the assumption that, in perpendicular direction to the nodes, the probability density falls off to zero sufficiently fast (essentially, faster than linearly). In a way this corresponds to (A.5), although our assumption has the drawback of not distinguishing between perpendicular and tangential properties of the density.

(iv) In what sense does the limit in (5) exist? Föllmer and Wakolbinger [17] have shown that if

$$
E\left[\int_{0}^{\tau} d t \mid b\left(Y_{t}\right)^{p}\right]<\infty
$$



for some $p \geqq 1$, then for allmost all $t \in[0, \tau]$ the limit $\lim _{\Delta t \downarrow 0} \frac{1}{\Delta t} E\left[X_{t+\Delta t}-X_{t} \mid \mathscr{F}_{t}\right]$
exists in $\mathscr{L}^{p}(\Omega, \mathscr{F}, P)$ and

$$
b\left(Y_{t}\right)=\lim _{\Delta t \downarrow 0} \frac{1}{\Delta t} E\left[X_{t+\Delta t}-X_{t} \mid \mathscr{F}_{t}\right]
$$

in the $\mathscr{L}^{p}$-sense.

\section{Application to Stochastic Mechanics}

It is the purpose of this section to explain how the diffusions constructed in the last section enter the theory of stochastic mechanics. To this end we will delineate its basic ideas.

Stochastic mechanics is an attempt at a probabilistic description of quantum phenomena $[18-20,12]$. It aims at dealing with quantum systems in classical terms. In agreement with other physical theories stochastic mechanics can be divided into two parts: the kinematics and the dynamics. The kinematical structure is given by associating a diffusion process $X_{t}$ governed by the SDE

$$
d X_{t}=b\left(X_{t}, t\right) d t+d w_{t}
$$

to the quantum motion in configuration space. The kinematical picture is completed by requiring that $X_{0}=\eta$ has a distribution identical to the quantum mechanical distribution at time 0 .

The dynamics specifies the influence of the potential $V$ on the motion. It should be a suitable generalization of classical dynamics. In stochastic mechanics this can either be achieved by a Newton law in the mean (Nelson $[19,20]$ ) or by a stochastic variational principle (Guerra, and Morato [21]; Nelson [12]). In this way the solution $\psi(x, t)$ of the Schrödinger equation

$$
i \hbar \partial_{t} \psi=\left[-\frac{\hbar^{2}}{2 m_{0}} \Delta+V(x)\right] \psi
$$

where $m_{0}$ denotes the mass and $\hbar$ is Planck's constant divided by $2 \pi$, is related to stochastic mechanics. In fact (provided $\psi$ is normalised),

$$
\varrho(x, t)=|\psi(x, t)|^{2},
$$

and therefore

$$
U=\left\{(x, t) \in \mathbb{R}^{d} \times \mathbb{R}_{+} \mid \psi(x, t) \neq 0\right\},
$$

and also

$$
b(x, t)=\frac{\hbar}{m}(\mathrm{Re}+\operatorname{Im}) \operatorname{grad} \ln \psi(x, t) \text { on } U .
$$

Moreover, the diffusion constant $v$ is equal to $\frac{\hbar}{2 m}$.

It is, of course, of vital interest to stochastic mechanics whether the diffusion processes postulated in this framework actually exist. To check that in typical cases the stochastic mechanical diffusions can be constructed it will be necessary to reformulate the conditions of Theorem 2 in terms of wave functions. We shall 
assume throughout this section that the wave functions are square-integrable and normalized.

The regularity conditions (A.1) and (A.2) can be reexpressed by

$$
\psi \in C^{2,1}\left(\mathbb{R}^{d} \times \mathbb{R}_{+}\right) .
$$

Denote by $\psi^{*}$ the complex conjugate of $\psi$. Then

$$
\begin{gathered}
\Delta \varrho=2\left[|\nabla \psi|^{2}+\operatorname{Re}\left(\psi^{*} \Delta \psi\right)\right], \\
\varrho \operatorname{div} v=2 v \operatorname{Im}\left[\psi^{*} \Delta \psi-\frac{1}{\varrho}\left(\psi^{*} \nabla \psi\right)^{2}\right],
\end{gathered}
$$

and the modulus of these expressions can be bounded in terms of $|\nabla \psi|^{2}$ and $\left|\psi^{*} \Delta \psi\right|$. Let $H^{m}\left(\mathbb{R}^{d}\right)$ be the $m$-th Sobolev space over $\mathbb{R}^{d}$. We have immediately the following

Theorem 3. Let (A.8) be satisfied. If

$$
\psi(\cdot, t) \in H^{2}\left(\mathbb{R}^{d}\right) \quad\left(\forall t \in \mathbb{R}_{+}\right),
$$

then (A.5) and (A.6) also hold.

Remark. (A.9) is, of course, a stronger condition than necessary because the proof of Theorem 2 depends only on the negative part of $v \Delta \varrho-\varrho \operatorname{div} v$. Note that (A.9) also implies $\|\operatorname{grad} \psi\|_{2}^{2} \in \mathscr{L}_{\mathrm{loc}}^{1}\left(\mathbb{R}_{+}\right)$, which is just the finite action condition (A.7).

Although the assumptions of Theorem 3 will be true in many quantum mechanical situations, it would be nice to have conditions in terms of the potential $V$ and the initial wave function $\psi_{0}=\psi(\cdot, 0)$. The stationary case, where $\psi(x, t)$ $=e^{-i t E / \hbar} \psi_{0}(x)$, is dealt with in (cf. [22, p. 54]).

Theorem 4. Let $\psi$ be a weak solution of

$$
\left(-\frac{\hbar^{2}}{2 m_{0}} \Delta+V\right) \tilde{\psi}=E \tilde{\psi},
$$

where $V$ is a measurable function $E$ a complex number. If

$$
V \in C^{m}\left(\mathbb{R}^{d}\right)
$$

then

$$
\psi \in C^{m-\left[\frac{d}{2}\right]+1}\left(\mathbb{R}^{d}\right)
$$

Here $\left[\frac{d}{2}\right]$ denotes the integer part of $\frac{d}{2}$.

So in the three-dimensional case $\psi$ has at least the same regularity as the potential. In particular, if $V \in C^{2}\left(\mathbb{R}^{3}\right)$ then (A.8) is satisfied.

To deal with the time-dependent situation we shall rely essentially on results by Wilcox [23]. The proof of the next theorem (as well as the proceeding one) depends on Sobolev's imbedding theorem [24].

Theorem 5. Let $m \geqq\left[\frac{d}{2}\right]+k+1, l \leqq k$ and $\psi \in C^{l}\left(\mathbb{R}_{+}, H^{m-l}\left(\mathbb{R}^{d}\right)\right.$ ). Then (a version of) $\psi$ has continuous derivatives up to order $k-l$ in space and $l$ in time simultaneously. 
If we note that $C^{2,1}=C^{2,0} \cap C^{0,1}$, then a twofold application of this theorem yields the important

Corollary 1. For $m \geqq\left[\frac{d}{2}\right]+3$,

$$
C^{0}\left(\mathbb{R}_{+}, H^{m}\left(\mathbb{R}^{d}\right)\right) \cap C^{1}\left(\mathbb{R}_{+}, H^{m-2}\left(\mathbb{R}^{d}\right)\right) \subset C^{2,1}\left(\mathbb{R}^{d} \times \mathbb{R}_{+}\right) .
$$

In particular, if $d=3$, then one has to make sure that the wave function satisfies

$$
\psi \in C^{0}\left(\mathbb{R}, H^{4}\left(\mathbb{R}^{3}\right)\right) \cap C^{1}\left(\mathbb{R}, H^{2}\left(\mathbb{R}^{3}\right)\right) .
$$

From the paper of Wilcox one can also read off conditions on the initial wave function $\psi_{0}$ and the potential $V$ such that (67) holds.

Theorem 6. Let $m \in \mathbb{N}$ and suppose that

$$
\psi_{0} \in H^{2 m}\left(\mathbb{R}^{d}\right),
$$

$V$ and its derivatives up to order $2 m-2$ are continuous and bounded in $\mathbb{R}^{d}$.

Then (a version of) the solution $\psi_{t}:=e^{-i t\left(-\frac{\hbar^{2}}{2 m_{0}} \Delta+v\right)} \psi_{0}$ of the Schrödinger equation satisfies

$$
\psi_{t} \in \bigcap_{l=0}^{m} C^{l}\left(\mathbb{R}_{+}, H^{2(m-l)}\left(\mathbb{R}^{d}\right)\right) .
$$

Self-adjointness of the Hamiltonian follows from Kato's theorem (since $V$ is bounded). Combining Theorem 3 with Corollary 1 and Theorem 6 we obtain

Theorem 7. If conditions (A.10) and (A.11) are satisfied for $2 m \geqq\left[\frac{d}{2}\right]+3$, then (A.5)
and (A.6) hold.

In particular, in the three-dimensional case $m$ has to be greater than or equal to two; i.e., we need that $\psi_{0} \in H^{4}\left(\mathbb{R}^{3}\right)$ and that $V$ and its derivatives up to order 2 are continuous and bounded.

Theorems 4 and 7 give conditions under which the stochastic mechanical diffusions exist, although the boundedness condition on the potential is physically unsatisfactory. In a given situation assumptions (A.10), (A.11) may not hold but (A.5) and (A.6) may be true nonetheless.

\section{Conclusion}

The results presented here show that a wide class of diffusion processes with singular drifts can be constructed. In particular, this is of interest in stochastic mechanics where the generic drifts are indeed singular. Moreover, both (global) existence and uniqueness are in the strong sense.

Conditions similar to (A.3), (A.5), and (A.6) are tantamount to all constructions of diffusions with singular drifts, and from a physical point of view they are not unreasonable. Our conditions are a kind of modification of the finite action condition of Carlen and Zheng. On the other hand, it does not seem to be within 
the scope of our method to relax the smoothness assumptions (A.1) and (A.2) considerably. As a slight modification we can replace the requirement of a derivative to exist by asking for a Lipshitz condition to be satisfied, which in turn will give uniform bounds, too.

The use of stopping times was essential to our analysis. An advantage of this is that the sample path behaviour enters in a transparent way. However, we did not make use of all information at our disposal: only $|\operatorname{grad} \ln \varrho| \operatorname{rather} \operatorname{than} \operatorname{grad} \ln \varrho$ entered the proofs; we also did not consider the direction of the drift $b$. It is clear that the unattainability of the nodes should follow only from conditions on the behaviour of these velocities perpendicular to the nodal set. Likewise global existence will involve the direction of $\operatorname{grad} \ln \varrho$ and $b$ at large distance from the origin (in $\mathbb{R}^{d}$ ).

It became obvious in the course of the proofs that the sample paths of the diffusion processes behave according to intuition with probability one. In terms of Itô calculus, however, one does not get control over all trajectories. So there exist trajectories which behave in a crazy manner, although their total probability is zero. From a physical point of view one would like to exclude this.

As a last remark it should be pointed out that our analysis carries over to the case where the state space is a Riemannian manifold.

Acknowledgements. The authors are grateful to E. A. Carlen, G.-F. Dell'Antonio, and W. A. Zheng for many constructive discussions. They would also like to thank W. A. Zheng for having critically read a previous draft of the manuscript.

\section{References}

1. Gihman, I.I., Skohorod, A.V.: Stochastic differential equations. Berlin, Heidelberg, New York: Springer 1972

2. Stroock, D.W., Varadhan, S.R.S.: Multidimensional diffusion processes. Berlin, Heidelberg, New York: Springer 1979

3. Ikeda, N., Watanabe, S.: Stochastic differential equations and diffusion processes. Amsterdam: North-Holland 1981

4. Albeverio, S., Høegh-Krohn, R.: A remark on the connection between stochastic mechanics and the heat equation. J. Math. Phys. 15, 1745 (1974)

5. Carmona, R.: Processus de diffusion gouverné par la forme de Dirichlet de l'opérateur de Schrödinger. In: Séminaire de probabilités XIII. Dellacherie, C. et al. (eds.). Berlin, Heidelberg, New York: Springer 1979

6. Nagasawa, M.: Segregation of a population in an environment. J. Math. Biol. 9, 213 (1980)

7. Albeverio, S., Fukushima, M., Karwowski, W., Streit, L.: Capacity and quantum mechanical tunneling. Commun. Math. Phys. 81, 501 (1981)

8. Albeverio, S., Blanchard, Ph., Høegh-Krohn, R.: Newtonian diffusions and planets, with a remark on non-standard dirichlet forms and polymers. In: Stochastic analysis and applications. Truman, A., Williams, D. (eds.). Lecture Notes in Mathematics, Vol. 1095. Berlin, Heidelberg, New York: Springer 1984

9. Fukushima, M.: Energy forms and diffusion processes. In: Mathematics + physics, lectures on recent results. Streit, L. (ed.). New York: World Scientific 1985

10. Blanchard, $\mathrm{Ph}$., Zheng, W.: Pathwise conservation law for stationary diffusion processes. In: Stochastic processes in classical and quantum systems. Albeverio, S. et al. (eds.). Lecture Notes in Physics. Berlin, Heidelberg, New York: Springer (to appear)

11. Nelson, E.: Critical diffusions. In: Séminaire de probabilités XIX. Azéma, J., Yor, M. (eds.). Berlin, Heidelberg, New York: Springer 1985 
12. Nelson, E.: Quantum fluctuations. Princeton, NJ: Princeton University Press 1985

13. Carlen, E.A.: Conservative diffusions. Commun. Math. Phys. 94, 293 (1984)

14. Zheng, W.A.: Tightness results for laws of diffusion processes. Application to stochastic mechanics. Ann. Inst. Henri Poincaré B 21, 103 (1985)

15. Zheng, W.A., Meyer, P.A.: Sur la construction de certaines diffusions. In: Séminaire de probabilités XX. Lecture Notes in Mathematics. Berlin, Heidelberg, New York: Springer (to appear)

16. Guerra, F.: Carlen processes: a new class of diffusions with sigular drifts. In: Quantum probability and applications II. Accardi, L., von Waldenfels, W. (eds.). Lecture Notes in Mathematics, Vol. 1136. Berlin, Heidelberg, New York: Springer 1985

17. Föllmer, H.: Time reversal on Wiener space. In: Stochastic processes - mathematics and physics (1st BiBoS-Symposium). Lecture Notes in Mathematics, Vol. 1158. Berlin, Heidelberg, New York: Springer 1986

18. Fürth, R.: Über einige Beziehungen zwischen klassischer Statistik und Quantenmechanik. Z. Physik 81, 143 (1933)

19. Nelson, E.: Derivation of the Schrödinger equation from Newtonian mechanics. Phys. Rev. 150, 1079 (1966)

20. Nelson, E.: Dynamical theories of Brownian motion. Princeton, NJ: Princeton University Press 1967

21. Guerra, F., Morato, L.M.: Quantization of dynamical systems and stochastic control theory. Phys. Rev. D 27, 1774 (1983)

22. Reed, M., Simon, B.: Methods of modern mathematical physics. Vol. II. Fourier analysis and self-adjointness. New York: Academic Press 1975

23. Wilcox, C.H.: Uniform asymptotic estimates for wave packets in the quantum theory of scattering. J. Math. Phys. 6, 611 (1965)

24. Sobolev, S.L.: Applications of functional analysis in mathematical physics, translations of mathematical monographs. Providence, RI: Am. Math. Soc. 1963

Communicated by A. Jaffe

Received February 20, 1986; in revised form October 20, 1986 
